\title{
Vitamin D Deficiency Predicts Poor Clinical Outcomes in Heart Failure Patients Undergoing Cardiac Resynchronization Therapy
}

\author{
P. Perge, A. M. Boros, L. Gellér, I. Osztheimer, Sz Szilágyi, T. Tahin, A. Apor, K. V. Nagy, \\ E. Zima $\mathbb{D}^{\mathrm{D}}$, L. Molnár, B. Merkely $\mathbb{D}$, and G. Széplaki
}

Heart and Vascular Center, Semmelweis University, Városmajor utca 68, Budapest 1122, Hungary

Correspondence should be addressed to B. Merkely; merkely.bela@kardio.sote.hu

Received 1 February 2019; Accepted 6 May 2019; Published 13 October 2019

Guest Editor: Agata Bielecka-Dabrowa

Copyright (C) 2019 P. Perge et al. This is an open access article distributed under the Creative Commons Attribution License, which permits unrestricted use, distribution, and reproduction in any medium, provided the original work is properly cited.

\begin{abstract}
Background and Aims. Resynchronization therapy (CRT) improves mortality and induces reverse remodeling in heart failure (HF) patients with reduced ejection fraction and wide QRS. Nonetheless, some patients do not improve despite the optimal medical therapy and right indications for device implantation. Therefore, finding biomarkers suitable for identification of those patients is crucial. Vitamin D plays a classic hormonal role in the regulation of bone metabolism and also has physiological functions in wide range of nonskeletal tissues. Based on recent studies, low levels of vitamin D seem to directly contribute to pathogenesis and worsening of HF. We planned to assess the role of vitamin D levels on clinical outcomes of HF patients undergoing CRT. Methods and Results. We enrolled $136 \mathrm{HF}$ patients undergoing CRT. Total plasma vitamin D levels were measured at baseline and 6 months later. Primary endpoint was 5-year all-cause mortality; secondary endpoint was lack of good clinical response, defined as less than $15 \%$ increase of left ventricular ejection fraction after six months. During follow-up, 58 patients reached the primary, and 45 patients reached the secondary endpoint. Vitamin D levels less than $24.13 \mathrm{ng} / \mathrm{mL}$ predicted 5-year mortality $(p=0.045)$ and poor clinical response $(p=0.03)$ after adjusting to all significant baseline predictors. Conclusion. Our study showed that vitamin D deficiency has a significant impact in heart failure patients; it is an independent predictor of lack of midterm clinical response and long-term mortality in patients undergoing CRT. Therefore, monitoring vitamin D status of heart failure patients could be of clinical significance.
\end{abstract}

\section{Introduction}

Heart failure (HF) bears a major public health impact with constantly growing incidence, despite continuous improvements in prevention, diagnosis, and therapy [1]. Cardiac resynchronization therapy (CRT) is an effective therapeutic option for symptomatic HF patients with severely reduced left ventricular ejection fraction (LVEF) and wide QRS. In most patients, mortality and morbidity are reduced, while functional capacity and HF symptoms are improved $[2,3]$. Despite the repeated refinements in the guidelines for optimal patient selection, poor clinical response to CRT is still prevalent [4], thus recognizing further predictors of outcome is crucial.

Vitamin D, initially known as a key hormone of bone metabolism, has several extraskeletal physiologic functions. Based upon recent studies, vitamin D is an important regula- tor of the renin-angiotensin-aldosterone system (RAAS), inflammatory cytokines, and extracellular matrix (ECM) turnover. Moreover, vitamin D deficiency directly contributes to pathogenesis of HF by the loss of above modulating mechanisms, causing remodeling of the heart [5]. Numerous cross-sectional and longitudinal studies showed that vitamin $\mathrm{D}$ deficiency is associated with increased risk of HF; in addition, worse prognosis of already diagnosed HF was also demonstrated [6]. Interestingly, vitamin D supplementation in primary or secondary prevention of $\mathrm{HF}$ is controversial; there is no definitive evidence supporting a favorable role of vitamin D supplementation [7].

There is limited data in the literature assessing the role of vitamin $\mathrm{D}$ deficiency in predicting clinical response to CRT. The results of previous small-scale studies suggest that patients with low levels of vitamin $\mathrm{D}$ show inadequate six-month clinical response to CRT $[8,9]$; however, no 
data exists regarding hard endpoints. The aim of our study was to determine the predictive value of vitamin $\mathrm{D}$ deficiency on the long-term mortality after CRT and confirm the association with poor midterm clinical response.

\section{Methods}

2.1. Study Population. 141 consecutive HF patients were enrolled to our prospective, single-center, observational study. The purpose of the study was to evaluate the prognostic value of various biomarkers in a cohort of $\mathrm{HF}$ patients previously described in details, including routine laboratory markers, uric acid, complement components, and novel HF biomarkers [10-14]. This present study focused on the role of vitamin $\mathrm{D}$ levels in the prognosis after CRT implantation.

We enrolled chronic heart failure patients with optimal medical therapy, symptomatic HF (New York Heart Association functional class II-IVa), left ventricular ejection fraction (LVEF) below 35\%, and wide QRS complex $(>120 \mathrm{msec})$ in the baseline electrocardiogram (ECG). Vitamin D supplementation was not included in the medical therapy before enrolment and during the follow-up. The patients underwent CRT implantation according to the current guidelines [15] in the Heart and Vascular Center of Semmelweis University, Budapest, between September 2009 and December 2010. Severe systemic inflammatory and hematologic diseases and active malignancies were considered as exclusion criteria; we excluded 4 patients based on these conditions; furthermore, we did not have complete dataset of one patient.

2.2. Clinical Endpoints and Follow-Up. The primary endpoint of the study was five-year all-cause mortality. Good clinical response, defined as an at least 15\% increase of LVEF after six months of CRT, was considered as the secondary endpoint. All patients gave written informed consent before enrolment to the study. The investigation conformed to the Declaration of Helsinki; the study protocol was approved by the local Ethics Committee.

The follow-up period lasted five years; we conducted visits at six months, two years, and five years after CRT implantation. Detailed physical examinations, laboratory tests, ECG, and echocardiography were performed in a total of 136 patients at baseline. At follow-up visits, functional status of the patients was evaluated by assessing the NYHA classification; their medical therapy and relevant adverse medical events were documented. Laboratory blood analyses, echocardiography, and ECG were repeated at six months.

2.3. Laboratory Measurements, Exposure to Sunlight, and Echocardiography. We obtained venous blood samples from the patients, afterwards processed the serum and ethylenediaminetetraacetic acid plasma aliquots within two hours after sampling. Samples were stored at $-80^{\circ} \mathrm{C}$ for later laboratory measurements. Total serum 25(OH)-vitamin D levels were measured with Roche Elecsys vitamin D total assay kits (Cat. No.: 05894913190, Roche Diagnostics, Mannheim, Germany. Reference value is $>30 \mathrm{ng} / \mathrm{mL}$ in the Central Laboratory of Semmelweis University, respectively). N-terminal of the prohormone brain natriuretic peptide (NT-proBNP) levels was measured using Roche Elecsys NT-proBNP II kits (Cat. No.: 04842464190, Roche Diagnostics, Mannheim, Germany) with a Cobas e 411 analyzer (Roche Diagnostics, Mannheim, Germany). Serum calcium levels were measured CA2 kits (Cat. No.: 05061482190, Roche Diagnostics, Mannheim, Germany) with a Cobas Integra 400 Plus analyzer (Roche Diagnostics, Mannheim, Germany).

Exposure to sunlight was assessed by the cumulative hours of sunshine in the 30-day preceding enrolment, based upon the public databases of the National Meteorological Service [16].

Echocardiography and offline measurements were carried out by licensed echocardiographic experts using a Phillips iE 33 system, Philips Xcelera R3.1.L1, and Philips Qlab 9.0 software. LVEF was calculated using Simpson's biplane method. The reproducibility of echocardiographic measurements was determined; interobserver and intraobserver variability was assessed with Lin's concordance correlation coefficient using 12-12 pair of sample data; substantial correlation was proven, as described previously in this cohort (interobserver variability: $\rho_{\mathrm{c}}=0.956(0.89-0.98)$; intraobserver variability: $\left.\rho_{\mathrm{c}}=0.96(0.89-0.97)\right)$.

2.4. Statistical Analysis. As the majority of the variables showed nonparametric distributions, the data were expressed as the medians with interquartile ranges or as percentages with the event numbers. Continuous variables were compared with the Wilcoxon matched pair test and the Mann-Whitney test, as appropriate. A chi-squared test was applied for categorical data comparisons. The Cox and univariate logistic regression analyses were applied to determine the baseline predictors of 5-year mortality and the lack of good clinical response; the continuous variables were standardized by one standard deviation increase for the regression analyses. We used receiver operating characteristic (ROC) analysis, and the continuous variables were dichotomized and then the Kaplan-Meier curves were compared using the log-rank tests. In the multivariable Cox regression and logistic regression models, the baseline model included variables with $p<0.1$ value from the univariate analysis, and further adjusted models were built in a forward stepwise manner.

In the present study, a two-tailed $p$ value of $<0.05$ was considered statistically significant. Statistical analyses were performed using IBM SPSS 22 (Apache Software Foundation, USA) and GraphPad Prism 6.03 (GraphPad Software, Inc., USA) software products.

\section{Results}

3.1. Baseline Characteristics and Effects of CRT on the Study Population. The baseline characteristics of the 136 patients are detailed in Table 1; the comparison of surviving patients and nonsurvivors is showed. Deceased patients had higher LVEF and NT-proBNP levels at baseline, while the proportion of patients with left bundle branch block (LBBB) and beta blocker therapy was significantly lower. Baseline plasma vitamin D level of the patients were $20.9 \mathrm{ng} / \mathrm{mL}$ (15.2-31.7), and 
TABLE 1: Baseline characteristics.

\begin{tabular}{|c|c|c|c|c|}
\hline Clinical variables & All patients $(n=136)$ & Surviving patients $(n=78)$ & Deceased patients $(n=58)$ & $p$ value \\
\hline Age (years) & $67(60-73)$ & $67(60-71)$ & $70(62-74)$ & 0.067 \\
\hline Gender (male) & $81(110)$ & $76(59)$ & $88(51)$ & 0.078 \\
\hline BMI $\left(\mathrm{kg} / \mathrm{m}^{2}\right)$ & $27(24-30)$ & $27(25-30)$ & $27(23-29)$ & 0.196 \\
\hline Ischemic HF & $57(78)$ & $53(41)$ & $64(37)$ & 0.201 \\
\hline LBBB & $82(112)$ & $91(71)$ & $71(41)$ & 0.003 \\
\hline CRT-D & $16(22)$ & $18(14)$ & $14(8)$ & 0.533 \\
\hline Opt. lead position & $74(100)$ & $73(57)$ & $74(43)$ & 0.896 \\
\hline QRS (msec) & $163(141-184)$ & $164(141-184)$ & $163(144-185)$ & 0.691 \\
\hline $\operatorname{LVEF}(\%)$ & $28(23-33)$ & $28(23-32)$ & $34(25-40)$ & $<0.001$ \\
\hline LVESV (mL) & $211(154-276)$ & $218(160-276)$ & $207(141-268)$ & 0.353 \\
\hline LVEDV (mL) & $303(251-361)$ & $313(251-382)$ & $299(242-343)$ & 0.448 \\
\hline NYHA III- IV & $86(117)$ & $86(117)$ & $91(53)$ & 0.138 \\
\hline Hypertension & $56(76)$ & $55(43)$ & $57(33)$ & 0.054 \\
\hline Hyperlipidemia & $24(33)$ & $22(17)$ & $28(16)$ & 0.423 \\
\hline Diabetes mellitus & $37(50)$ & $33(26)$ & $41(24)$ & 0.339 \\
\hline ACEi/ARB & $96(130)$ & $97(76)$ & $93(54)$ & 0.277 \\
\hline $\mathrm{BB}$ & $90(122)$ & $95(74)$ & $83(48)$ & 0.022 \\
\hline MRI & $71(96)$ & $74(58)$ & $65(33)$ & 0.258 \\
\hline Calcium (mmol/L) & $2.43(2.34-2.49)$ & $2.43(2.36-2.49)$ & $2.41(2.32-2.50)$ & 0.922 \\
\hline NT-proBNP (pg/mL) & $2612(1377-5124)$ & $2101(1000-3555)$ & 4035 (2125-6479) & $<0.001$ \\
\hline Sunlight (hours) & $156(67-241)$ & $157(102-241)$ & $148(66.7-221)$ & 0.748 \\
\hline
\end{tabular}

Data is expressed as median with interquartile range for continuous variables and as percentage with event numbers for categorical variables. BMI: body mass index; ischemic HF: ischemic etiology of the heart failure; LBBB: left bundle branch block; CRT-D: cardiac resynchronization therapy with implantable cardioverter defibrillator; Opt. lead position: lateral or posterolateral left ventricular lead position; LVEF: left ventricular ejection fraction; LVESV: left ventricular end systolic volume; LVEDV: left ventricular end diastolic volume; NYHA III-IV: New York Heart Association classification 3-4; ACEi/ARB: angiotensin convertase inhibitor/angiotensin receptor blocker therapy; BB: beta blocker therapy; MRI: mineralocorticoid receptor inhibitor therapy; NT-proBNP: N-terminal of the prohormone brain natriuretic peptide, sunlight: cumulative duration of sunlight in the 30 days prior enrolment.

we observed no significant change after six months of CRT (6 months: $21.5(16.2-28.3), p=0.43)$. The study population was described in detail previously; LVEF and left ventricular endsystolic and end-diastolic volumes (LVESV and LVEDV) decreased significantly [14].

3.2. Association of Baseline Vitamin D Concentrations with Clinical Outcomes. 58 patients (43\%) died during the 5-year follow-up; those who survived had significantly higher baseline vitamin D levels at baseline (23.07 (16.58-31.73) vs. 18.3 (13.81-23.75) ng/mL, $p=0.018)$. In 45 patients (33\%), we observed the lack of good clinical response six months after CRT implantation; similarly, we measured increased baseline vitamin $\mathrm{D}$ levels in patients with good clinical response (22.56 (15.6-31.87) vs. $18.12(13.95-23.43) \mathrm{ng} / \mathrm{mL}$, $p=0.027)$.

To establish an optimal cut-point for the further assessment of the clinical outcomes, we used receiver operating characteristic analysis. Plasma vitamin D below $24.13 \mathrm{ng} / \mathrm{mL}$ seemed to be an optimal cut-point for 5-year mortality (AUC $=0.62$ (0.52-0.71), $p=0.018$; sensitivity: $78 \%(65-87)$; specificity: $45 \%(34-58))$ and lack of 6-month clinical response $(\mathrm{AUC}=0.62(0.52-0.71), p=0.027$; sensitivity: $80 \%$ (65-90); specificity: $42 \%$ (32-53)), respectively (Figure 1).
Next, we created groups of patients with baseline vitamin D below and over $24.13 \mathrm{ng} / \mathrm{mL}$. The use of beta blockers was the only parameter that differed significantly between the subgroups; almost all patients in the group with higher vitamin D levels were on beta blockers (98\% vs. $85 \%, p=0.015)$. Sunlight exposure before enrolment did not differ significantly between groups $(p=0.56)$. When analyzing the severity of heart failure, there was no difference between groups at baseline reflected by the NT-proBNP levels (2626 (1683-5214) vs. 2518 (988-4791), $p=0.18)$ or the NYHA class $(2.97 \pm 0.48$ vs. $2.91 \pm 0.57$, $p=0.61)$. NT-proBNP levels remained significantly elevated only in the subgroup of patients with low vitamin $\mathrm{D}$ levels (1216 (337-2214) vs. $2116 \quad(927-3865) \quad p=0.019)$ (Figure 2). Moreover, their functional status (NYHA class) also did not improve $(1.9 \pm 0.42$ vs. $2.21 \pm 0.5, p=0.001)$ (Figure 3).

\subsection{Predictors of 5-Year Mortality and Lack of Good Clinical} Response. We analyzed the 5-year all-cause mortality by univariate Cox regression and the six-month clinical response using univariate logistic regression analysis. Vitamin D level lower than $24.13 \mathrm{ng} / \mathrm{mL}$ was significantly associated with increased risk of long-term mortality (HR 2.25 (1.21-4.17), 


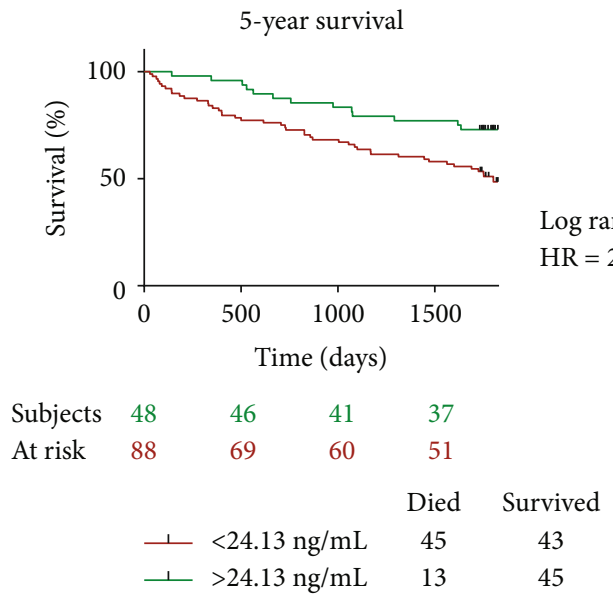

(a)
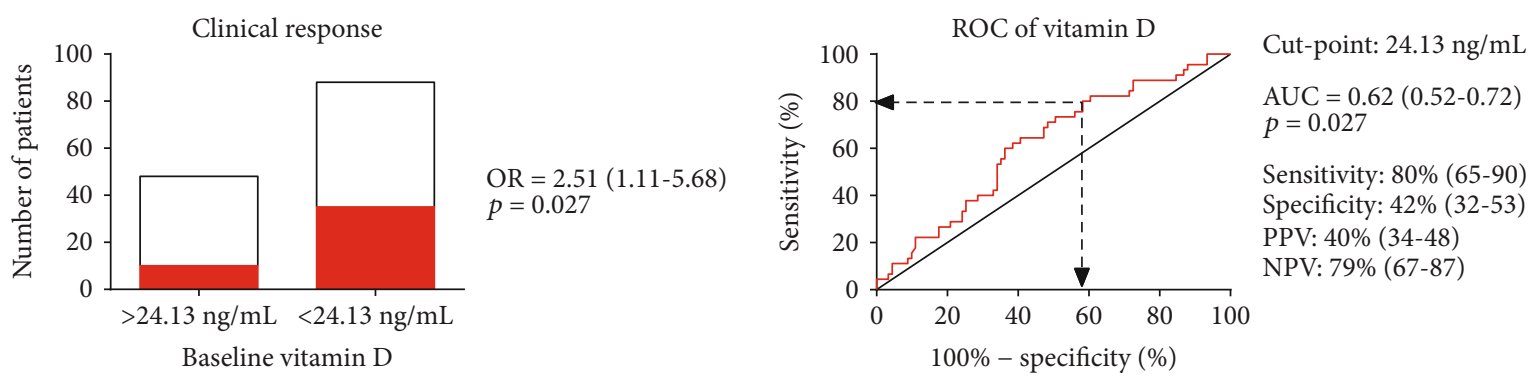

Clinical response

No clinical response

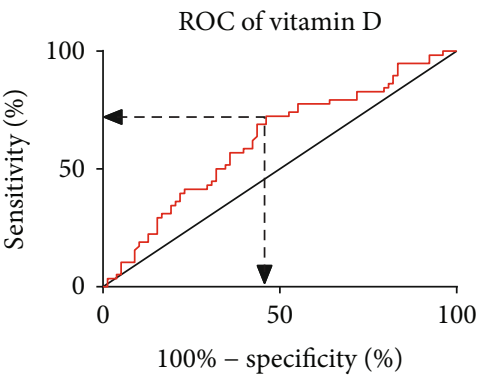

Cut-point: $24.13 \mathrm{ng} / \mathrm{mL}$

AUC $=0.62(0.52-0.71)$

$p=0.018$

Sensitivity: 78\% (65-87)

Specificity: $45 \%$ (34-57)

PPV: $51 \%$ (40-62)

NPV: $73 \%(58-85)$

$100 \%$ - specificity (\%)

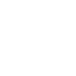$$
\text { . }
$$ 

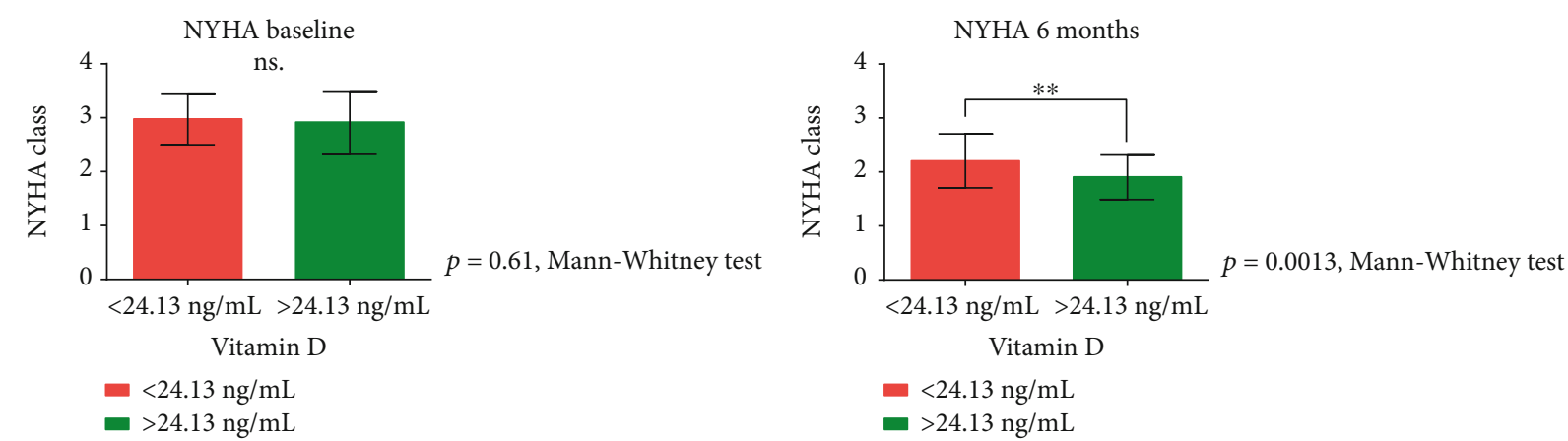

FIGURE 3: Severity of heart failure at baseline and after six months of CRT. We compared the baseline and six-month severity of heart failure using NYHA classification in patient groups of baseline plasma vitamin D levels below and above $24.13 \mathrm{ng} / \mathrm{mL}$ using the Mann-Whitney test. CRT: cardiac resynchronization therapy; NYHA class: classification of heart failure according to the New York Heart Association.

of NT-proBNP $(p=0.22)$. The detailed results of univariate logistic and Cox regression statistical analyses are showed in Supplementary Table 1.

To determine the independent influence of decreased vitamin D levels on mortality, we set up a basic multivariable Cox regression model with the baseline clinical variables shown to be relevant by the univariate analysis $(p<0.10)$. Thus, the baseline multivariable model included age, LBBB, use of beta blocker therapy, and baseline NT-proBNP. In the following step, we entered vitamin $\mathrm{D}$ levels into the baseline model in a forward stepwise way. Vitamin D levels under $24.13 \mathrm{ng} / \mathrm{mL}$ predicted mortality in the multivariable model as well ( $\mathrm{HR}=1.92(1.02-1.45), p=0.045)$.

We use the same method to investigate the clinical response. We included the relevant factors to the basic multivariable model: hypertension, hyperlipidemia, mineralocorticoid receptor inhibitor therapy, and increasing levels of NT-proBNP. We entered vitamin D levels into the baseline model in a forward stepwise way. Similar to mortality prediction, vitamin D was an independent predictor of lack of good clinical response $(\mathrm{OR}=2.62 \quad(1.01-6.25), p=0.03)$. The detailed results of multivariate logistic and Cox regression statistical analyses are showed in Supplementary Table 2.

\section{Discussion}

4.1. Synopsis of Key Findings. Vitamin D levels under $24.13 \mathrm{ng} / \mathrm{mL}$ predicted long-term mortality and poor clinical response in HF patients undergoing CRT independently of all relevant baseline predictors including NT-proBNP. Furthermore, patients with vitamin D insufficiency had significantly higher NT-proBNP levels and suffered from more severe HF six months after CRT.

4.2. Possible Mechanisms and Explanation. Chronic HF is an emerging disease in the high- and middle-income countries, affecting millions of people and requiring considerable amount of healthcare expenditure. HF development is considered a compound pathophysiological process, involving the activation of neurohormonal and inflammatory pathways, tissue remodeling. Wide range of pharmaceutical and device-based treatment is available, yet the overall outcome of HF is still poor [17].
Vitamin D has been considered the key regulator of calcium and phosphorus homeostasis and bone mineralization. Besides the regulation of bone metabolism, recent studies showed that vitamin D has numerous extraskeletal functions. It plays various regulatory roles in several mechanisms considered fundamental in development of HF [18]. Vitamin D suppresses the expression of renin and RAAS activity [19] and modulates the turnover of the ECM by enhancing the production of matrix metalloproteinase inhibitors [20], and experimental studies showed that it promotes myocyte contraction and relaxation by modulating the calcium influx [21]. In case of vitamin D deficiency, with the loss of the previous regulating effects, hypertrophy, ECM deposition, and myocardial fibrosis may arise.

Clinical studies also confirmed that vitamin D has a strong impact in HF. The risk of developing HF was increased in longitudinal studies in case of low levels of vitamin $\mathrm{D}$, while prevalent vitamin $\mathrm{D}$ deficiency was found among HF patients [6]. Furthermore, it was associated with significantly worse prognosis $[22,23]$.

Although vitamin $\mathrm{D}$ deficiency contributes to the development of HF by several described regulatory mechanisms, the clear beneficial effect of vitamin D supplementation in HF patients is still under debate, based upon recent randomized studies [24, 25]. Further randomized studies with consistent enrolment criteria are needed to validate the benefit.

Previous small studies also suggested that low levels of vitamin $\mathrm{D}$ predict poor 6-month response after CRT implantation $[8,9]$, yet there was no data available regarding longterm mortality. In our study, we also confirmed the role of vitamin $\mathrm{D}$ in the prediction of the lack of good clinical response after CRT. Baseline vitamin D levels under $24.13 \mathrm{ng} / \mathrm{mL}$ were significantly associated with poor clinical response, independently of all relevant baseline predictors. Patients with lower baseline vitamin D levels had 2.5-fold risk of lack of good clinical response after CRT.

Furthermore, we demonstrated that reduced baseline vitamin D levels were significant predictors of 5-year mortality after CRT; the mortality risk was independent of all relevant baseline predictors. Patients with vitamin D levels under $24.13 \mathrm{ng} / \mathrm{mL}$ had more than 2 -fold mortality risk during the follow-up. 
We used the NYHA classification of HF and NT-proBNP levels as surrogate markers to further assess the clinical response of patients. Interestingly, at baseline there were no significant differences between groups of patients with vitamin D levels below and above $24.13 \mathrm{ng} / \mathrm{mL}$. After six months of CRT, patients with reduced vitamin D levels had significantly higher NT-proBNP levels and NYHA classes, indicating a more severe stage of $\mathrm{HF}$, enhanced progression, and poor clinical response.

According to the 2011 classification by the Institute of Medicine, 25(OH)-vitamin D levels below $20 \mathrm{ng} / \mathrm{mL}$ are considered inadequacy, while levels between 20 and $50 \mathrm{ng} / \mathrm{mL}$ are considered adequacy [26]. Our statistically verified cutpoint reaching almost the limit of vitamin $\mathrm{D}$ inadequacy also supports the previous findings regarding the close association of low vitamin D levels and poor clinical outcomes in HF.

4.3. Limitations. Remarkable limitations of our study are the relatively small sample size and the single-center design. Allcause mortality was considered as the primary endpoint; cause of death was not investigated separately in this analysis due to the relatively low event numbers. The assessment of the sunlight exposure of patients can only credited as an approximate, since the average cumulative hours of sunshine in Hungary were calculated. Our results can be represented as hypothesis-generating findings; this observation does not prove direct causality between vitamin D levels and prognosis in HF patients undergoing CRT. Validation with prospective, multicenter studies is necessary.

\section{Conclusion}

Our results further verify the previous findings concerning the association of reduced vitamin $\mathrm{D}$ levels with the poor prognosis in various HF populations. Decreased baseline vitamin D levels predicted poor outcomes after CRT, functional status, and clinical response. Long-term survival of the patients was significantly worse compared with patients with adequate baseline vitamin $\mathrm{D}$ levels. Therefore, assessing the vitamin $\mathrm{D}$ homeostasis of $\mathrm{HF}$ patients before CRT implantation might be helpful in identifying the high-risk patients, among whom nonresponse should be anticipated.

\section{Data Availability}

The data used to support the findings of this study are available from the corresponding author upon request.

\section{Disclosure}

A previous version of the manuscript has been published as an abstract in the EP Europace Supplements following the Cardiostim EHRA Europace 2016 Congress (poster presentation no. 001986).

\section{Conflicts of Interest}

The authors declare that there is no conflict of interest regarding the publication of this article.

\section{Authors' Contributions}

Merkely B. and Széplaki G. contributed equally to the work and both should be regarded as last authors of the article.

\section{Acknowledgments}

Our observational study was supported in the form of unrestricted research grants by the Hungarian Foundation Programs "Semmelweis Egyetem Híd Projekt" (Grant: TÁMOP-4.2.2-08/1/KMR-2008-0004), "Semmelweis Egyetem Magiszter Program” (Grant: TÁMOP-4.2.2./B10/1.210-0013), Hungarian Scientific Research Fund (Grant: OTKA K 105555), “Az orvos-, egészségtudományi- és gyógyszerészképzés tudományos mühelyeinek fejlesztése” (Grant: EFOP-3.6.3-VEKOP-16-2017-00009), and János Bolyai Research Scholarships of the Hungarian Academy of Sciences.

\section{Supplementary Materials}

Supplementary Tables 1 and 2: the detailed results of logistic and Cox regression statistical analyses are presented, including both univariate and multivariate models. (Supplementary Materials)

\section{References}

[1] E. Braunwald, "Heart failure," JACC: Heart Failure, vol. 1, no. 1, pp. 1-20, 2013.

[2] J. G. Cleland, J. C. Daubert, E. Erdmann et al., "The effect of cardiac resynchronization on morbidity and mortality in heart failure," New England Journal of Medicine, vol. 352, no. 15, pp. 1539-1549, 2005.

[3] A. S. Tang, G. A. Wells, M. Talajic et al., "Cardiac-resynchronization therapy for mild-to-moderate heart failure," New England Journal of Medicine, vol. 363, no. 25, pp. 2385-2395, 2010.

[4] Q. Zhang, Y. Zhou, and C. M. Yu, "Incidence, definition, diagnosis, and management of the cardiac resynchronization therapy nonresponder," Current Opinion in Cardiology, vol. 30, no. 1, pp. 40-49, 2015.

[5] V. Rai and D. K. Agrawal, "Role of vitamin D in cardiovascular diseases," Endocrinology and Metabolism Clinics of North America, vol. 46, no. 4, pp. 1039-1059, 2017.

[6] C. D'Amore, F. Marsico, A. Parente et al., "Vitamin D deficiency and clinical outcome in patients with chronic heart failure: a review," Nutrition, Metabolism and Cardiovascular Diseases, vol. 27, no. 10, pp. 837-849, 2017.

[7] W. L. Jiang, H. B. Gu, Y. F. Zhang, Q. Q. Xia, J. Qi, and J. C. Chen, "Vitamin D supplementation in the treatment of chronic heart failure: a meta-analysis of randomized controlled trials," Clinical Cardiology, vol. 39, no. 1, pp. 56-61, 2016.

[8] H. Sunman, A. Özkan, H. Yorgun et al., "Vitamin D levels predict the response to cardiac resynchronization therapy in patients with systolic heart failure," Turk Kardiyoloji Dernegi Arsivi-Archives of the Turkish Society of Cardiology, vol. 44, no. 8, pp. 670-676, 2016. 
[9] A. Separham, L. Pourafkari, B. Kazemi et al., "Vitamin D deficiency and functional response to CRT in heart failure patients," Herz, vol. 44, no. 2, pp. 147-154, 2019.

[10] A. M. Boros, P. Perge, Z. Jenei et al., "Measurement of the red blood cell distribution width improves the risk prediction in cardiac resynchronization therapy," Disease Markers, vol. 2016, Article ID 7304538, 13 pages, 2016.

[11] A. M. Boros, G. Széplaki, P. Perge et al., "The ratio of the neutrophil leucocytes to the lymphocytes predicts the outcome after cardiac resynchronization therapy," Europace, vol. 18, no. 5, pp. 747-754, 2016.

[12] G. Széplaki, A. M. Boros, S. Szilágyi et al., "Complement C3a predicts outcome in cardiac resynchronization therapy of heart failure," Inflammation Research, vol. 65, no. 12, pp. 933-940, 2016.

[13] A. M. Boros, P. Perge, K. V. Nagy et al., "The impact of cardiac resynchronization therapy on routine laboratory parameters," Interventional Medicine and Applied Science, vol. 9, no. 1, pp. 1-8, 2017.

[14] P. Perge, A. M. Boros, S. Szilágyi et al., "Novel biomarkers in cardiac resynchronization therapy: hepatocyte growth factor is an independent predictor of clinical outcome," Revista Española de Cardiología (English Edition), vol. 72, no. 1, pp. 48-55, 2019.

[15] P. E. Vardas, A. Auricchio, J. J. Blanc et al., "Guidelines for cardiac pacing and cardiac resynchronization therapy: the task force for cardiac pacing and cardiac resynchronization therapy of the European Society of Cardiology. Developed in collaboration with the European Heart Rhythm Association," European Heart Journal, vol. 28, no. 18, pp. 2256-2295, 2007.

[16] https://www.met.hu/eghajlat/magyarorszag_eghajlata/eghajlati_ adatsorok/Budapest/adatok/napi_adatok/.

[17] E. Tanai and S. Frantz, "Pathophysiology of heart failure," Comprehensive Physiology, vol. 6, no. 1, pp. 187-214, 2015.

[18] A. Pourdjabbar, G. Dwivedi, and H. Haddad, "The role of vitamin D in chronic heart failure," Current Opinion in Cardiology, vol. 28, no. 2, pp. 216-222, 2013.

[19] Y. C. Li, J. Kong, M. Wei, Z. F. Chen, S. Q. Liu, and L. P. Cao, "1,25-Dihydroxyvitamin $\mathrm{D}_{3}$ is a negative endocrine regulator of the renin-angiotensin system," The Journal of Clinical Investigation, vol. 110, no. 2, pp. 229-238, 2002.

[20] K. T. Weber, W. B. Weglicki, and R. U. Simpson, "Macro- and micronutrient dyshomeostasis in the adverse structural remodelling of myocardium," Cardiovascular Research, vol. 81, no. 3, pp. 500-508, 2009.

[21] R. U. Simpson, S. H. Hershey, and K. A. Nibbelink, "Characterization of heart size and blood pressure in the vitamin D receptor knockout mouse," The Journal of Steroid Biochemistry and Molecular Biology, vol. 103, no. 3-5, pp. 521-524, 2007.

[22] I. Gotsman, A. Shauer, D. R. Zwas et al., "Vitamin D deficiency is a predictor of reduced survival in patients with heart failure; vitamin $\mathrm{D}$ supplementation improves outcome," European Journal of Heart Failure, vol. 14, no. 4, pp. 357-366, 2012.

[23] D. Gruson, B. Ferracin, S. A. Ahn et al., "1,25-Dihydroxyvitamin D to PTH(1-84) ratios strongly predict cardiovascular death in heart failure," PLoS One, vol. 10, no. 8, article e0135427, 2015.

[24] K. K. Witte, R. Byrom, J. Gierula et al., "Effects of vitamin D on cardiac function in patients with chronic HF: the VINDICATE study," Journal of the American College of Cardiology, vol. 67, no. 22, pp. 2593-2603, 2016.

[25] A. Zittermann, J. B. Ernst, S. Prokop et al., "Vitamin D supplementation and bone turnover in advanced heart failure: the EVITA trial," Osteoporosis International, vol. 29, no. 3, pp. 579-586, 2018.

[26] A. C. Ross, J. A. E. Manson, S. A. Abrams et al., "The 2011 report on dietary reference intakes for calcium and vitamin $\mathrm{D}$ from the Institute of Medicine: what clinicians need to know," The Journal of Clinical Endocrinology \& Metabolism, vol. 96, no. 1, pp. 53-58, 2011. 


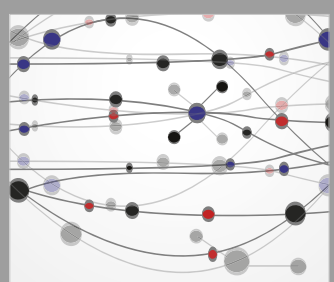

The Scientific World Journal
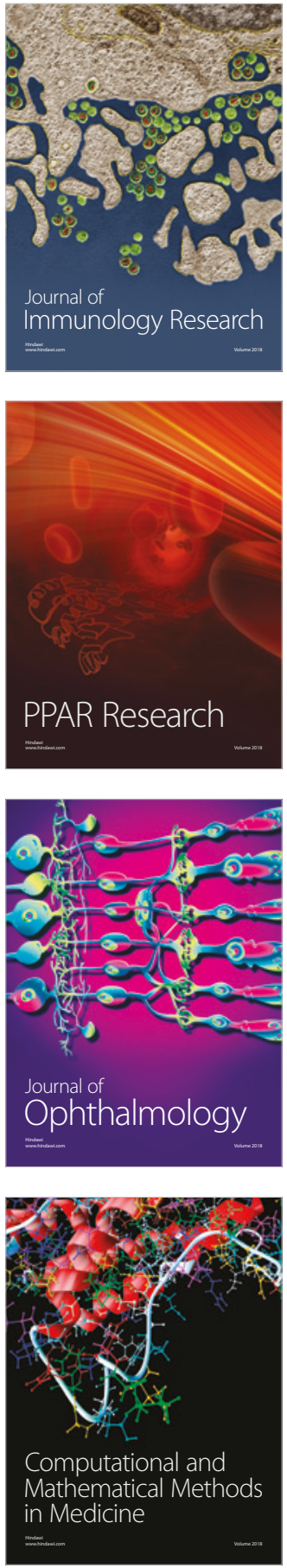

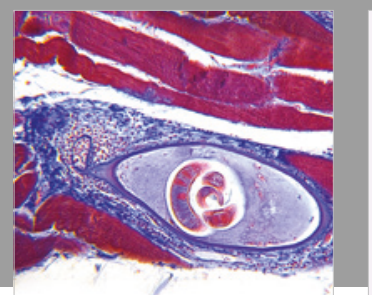

Gastroenterology Research and Practice

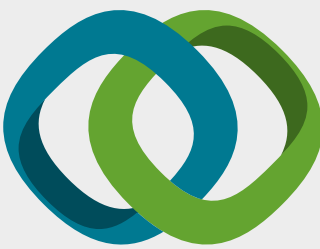

\section{Hindawi}

Submit your manuscripts at

www.hindawi.com
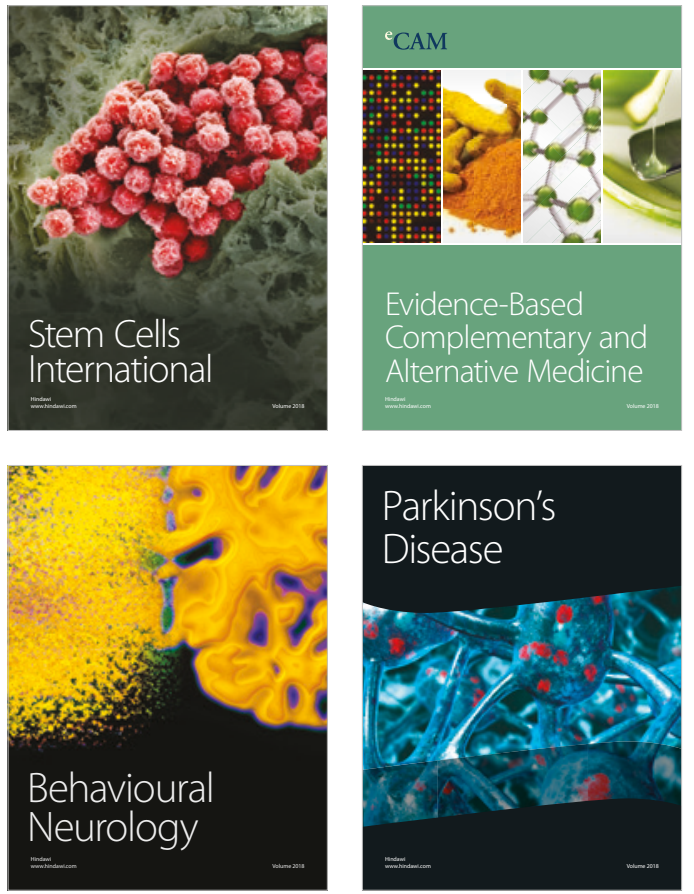

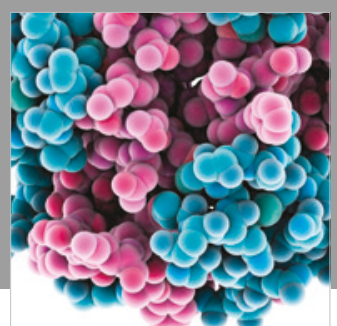

ournal of

Diabetes Research

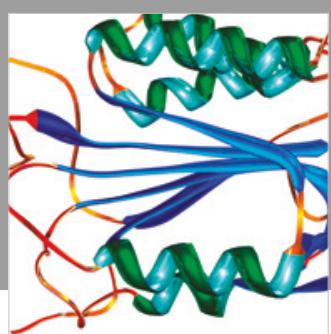

Disease Markers
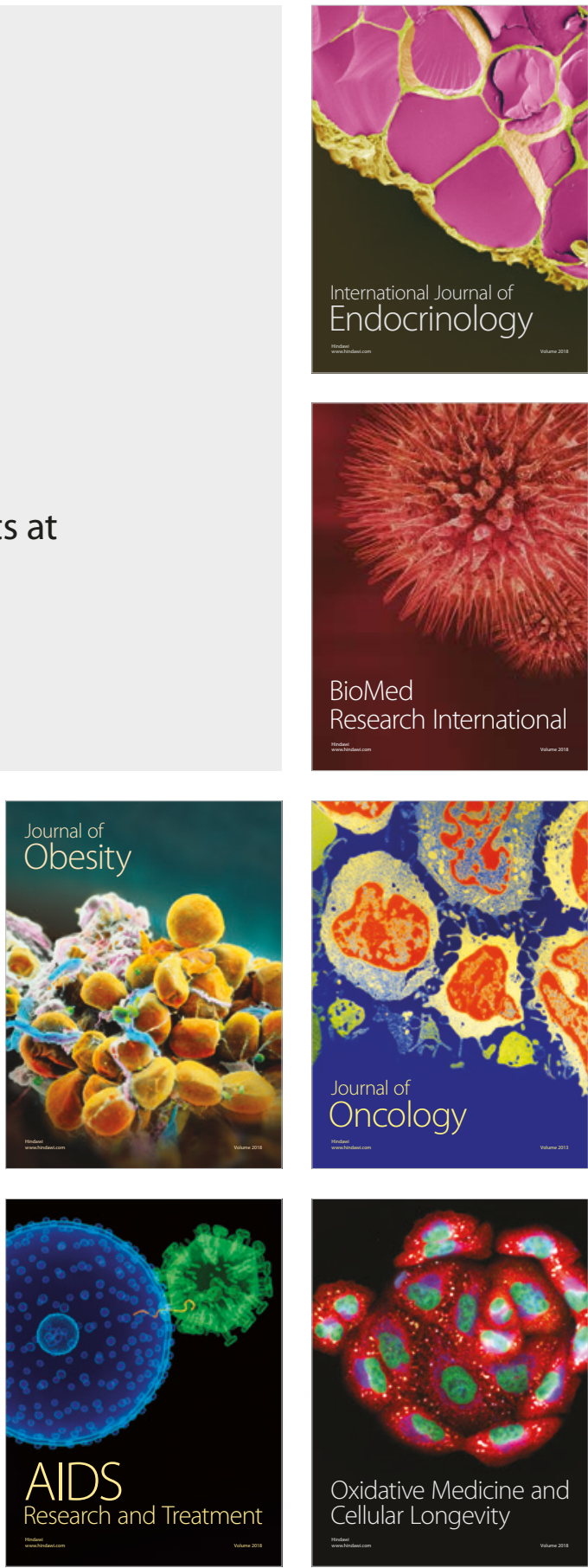